\title{
Author Index Vol. 63, No. 2,1995
}

Ehlers,W. 124 Freyberger, H.J. 61,63,90 Hettinger, R. 124 Heuft,G. 63 Janssen, P.L. 63

Jantschek, G. 112 Malchow, C.P. 90 Mezzich, J.E. 71 Muhs,A. 99, 112

Öri,C. 99 Paar,G. 124 Rodewig,K. 112 Schmolke, M.M. 71 Schneider, W. 61,63,90 Schüssler,G 81 Stieglitz, R.-D. 81 Wietersheim, J. von 112

Subject Index Vol. 63, No. 2,1995

Assessment instruments 81 Borderline syndrome 99 Comorbidity 90 Comprehensive diagnosis

71 Defense mechanism 124 Diagnostic concepts 63

Criteria for Research 90

instruments 81 DSM-ÏV 71 Factitious disorder 99 ICD-10 63,71,90 Idiographic and cultural

formulations 71

Interrater reliability 90

Kappa 90

Multiaxial approaches 63

diagnosis 71

d. diagnostic system 112 Multidimensional assessment 81 Multimodal diagnosis 63 Multiple diagnoses 90 Operational diagnosis 90 Personality disorders 99 Psychosomatic disorders 90, 112 Psychotherapy planning 71 Rater variables 90 